ISSN: 2386-3919

ISSN electrónico: 2386-3927

DOI: http://dx.doi.org/10.14201/et20153324358

\title{
EL APRENDIZAJE-SERVICIO Y LAS COMUNIDADES DE APRENDIZAJE: DOS PROYECTOS ESCOLARES INNOVADORES QUE SE ENRIQUECEN MUTUAMENTE
}

\author{
Service-learning and learning communities: two \\ innovative school projects that are mutually enriched
}

\author{
Apprentissage par le service et les communautés \\ d'apprentissage: deux projets d'école innovante qui \\ s'enrichissent mutuellement
}

Carmen Álvarez Álvarez* y Gonzalo Silió SÁIZ***

* Departamento de Educación. Universidad de Cantabria. Asesora oficial de CdA en Cantabria

* CEIP Verdemar. ApS Cantabria

Recibido: 24-07-2014; Aceptado: 19-01-2015; Publicado: 30-10-2015

BIBLID [2386-3927 (2015) 33, 2; 43-58]

Ref. Bibl. CARMEN ÁLVAREZ ÁLVAREZ y GONZALO SILIÓ SÁIZ. El aprendizajeservicio y las comunidades de aprendizaje: dos proyectos escolares innovadores que se enriquecen mutuamente. Enseñanza \& Teaching, 33, 2-2015, 43-58.

RESUMEN: En este artículo se reflexiona sobre las interrelaciones que existen entre dos proyectos educativos de actualidad: el aprendizaje-servicio (ApS) y las comunidades de aprendizaje (CdA). El ApS es una metodología educativa aplicada mundialmente donde en un solo proyecto se conjuga un aprendizaje basado en la experiencia con la realización de un servicio solidario a la comunidad. CdA es un proyecto de transformación de la escuela para conseguir que la sociedad de la 
CARMEN ÁLVAREZ ÁLVAREZ Y GONZALO SILIÓ SÁIZ EL APRENDIZAJE-SERVICIO Y LAS COMUNIDADES DE APRENDIZAJE: DOS PROYECTOS ESCOLARES INNOVADORES QUE SE ENRIQUECEN MUTUAMENTE

información no excluya a ninguna persona, constituyendo una realidad en más de ciento noventa escuelas repartidas entre España e Iberoamérica. Entre ambos es posible mostrar diferencias, sobre todo en lo que se refiere a sus sustratos teóricos e intencionalidades pedagógicas y sociales, pero en las prácticas reales en la enseñanza que se desarrollan en centros educativos hay más que cierta sintonía, particularmente en la relación tan estrecha que cultivan ambos proyectos con la comunidad escolar. Por todo ello, concluimos que el aprendizaje-servicio y las comunidades de aprendizaje pueden presentarse como dos proyectos innovadores y relevantes que pueden enriquecerse mutuamente: pues para ambos es fundamental la aproximación escuela-comunidad-entorno y el voluntariado.

Palabras clave: aprendizaje-servicio; comunidades de aprendizaje; comunidad educativa; solidaridad; diálogo; aprendizaje.

SUMMARY: This article reflects on the interrelationships that exist between two educational projects of today: service-learning (ApS) and learning communities (CdA). The ApS is an educational methodology applied worldwide where a single project combines a learning based on experience with the implementation of a service to the community. CdA is a school transformation project to achieve that the information society does not exclude any person, constituting a reality in more than one hundred and ninety schools in Spain and Latin America. Between the two, it is possible to show differences, especially in what refers to its theoretical substrates, but in actual teaching practice in schools there is some harmony, particularly in the so closely that they cultivate both projects with the school community. Therefore, we conclude that service-learning and learning communities can occur as two innovative and relevant today projects which can be mutually enriching: because for both the approach school-community-environment and volunteering is essential.

Key words: service-learning; learning community; school community; solidarity; dialogue; learning.

RESUMÉ: Cet article reflète sur les interrelations qui existent entre les deux projets éducatifs d'aujourd'hui: apprentissage par le service (ApS) et les communautés d'apprentissage (CdA). L'ApS est une méthodologie pédagogique appliquée partout dans le monde où un projet unique combine un apprentissage basé sur l'expérience de la mise en ouvre d'un service à la communauté. CdA est un projet de transformation de l'école pour obtenir que la société de l'information n'exclut pas une personne, qui constitue une réalité dans les écoles de plus de cent quatre-vingt-dix en Espagne et en Amérique latine. Entre les deux, il est possible de montrer les différences, en particulier en ce qui se réfère à ses substrats théoriques et les intentions sociales et pédagogiques, mais dans les pratiques réelles dans l'enseignement dans les écoles il y a une harmonie, particulièrement dans le si étroitement qu'ils cultivent les deux projets avec la communauté scolaire. Par conséquent, nous concluons que l'apprentissage par le service et les communautés d'apprentissage, peuvent se produire comme deux projets innovants et pertinents aujourd'hui qui peuvent s'enrichir 
mutuellement: parce que la démarche de l'école-est essentielle pour l'environnement et le bénévolat.

Mots clés: service-apprentissage; communautés d'apprentissage; communauté éducative; solidarité; dialogue; apprentissage.

\section{INTRODUCCIÓN}

En el ámbito académico hay líneas de investigación educativa que se van gestando en base a concepciones diferentes que, en ocasiones (menos de lo deseable lamentablemente), derivan en propuestas prácticas y comparten contextos. Tal es el caso de la metodología del aprendizaje-servicio (ApS) (Furco y Billig, 2002; Tapia, 2006; Puig y Palos, 2006; Martín y otros, 2010; García Rodicio y Silió Sáiz, 2012) y el proyecto comunidades de aprendizaje (CdA) (Flecha y Puigvert, 2002; Elboj, Puigdellívol, Soler y Valls, 2006; Valls, 2006; VV. AA, 2010), que, si bien no son mayoritarias, en la actualidad empiezan a estar cada vez más presentes en nuestros contextos. Aunque son proyectos de diferente envergadura (por su trayectoria, calado, etc.) tiene sentido ponerlos en relación y ver en qué aspectos son alternativos y en cuáles complementarios, tratando de buscar la convergencia entre ambas propuestas y promover la mejora escolar. Hoy día ya hay docentes implicados en alguna de estas prácticas pedagógicas que se plantean cuestiones como las abordadas en el presente artículo e incluso hay CdA que participan en prácticas de ApS y centros que practicando ApS se plantean transformarse en CdA (García Pérez, 2012).

Ambos proyectos cuentan con fundamentos teóricos dispares y persiguen finalidades últimas diferentes, desarrollando, en consecuencia, prácticas de enseñanza-aprendizaje también distintas. Este trabajo no pretende ofrecer una revisión exhaustiva de ambos postulados pedagógicos, sino reflexionar sobre las posibilidades de encuentro entre los dos proyectos, para ver si la distancia que existe entre ellos es notable e insalvable o si por el contario hay una cercanía que podría beneficiar a aquellos docentes implicados en la mejora escolar de sus centros.

Para abordar este reto, analizaremos las fundamentaciones teóricas en las que se apoyan ambos proyectos, partiendo de que ambos realizan un esfuerzo por relacionar al centro con su comunidad educativa (Billig y Conrad, 1997, 1999; Weiler y otros, 1999; Melchior, 1999; Kingland y otros, 1995; Kinsley, 1997), lo que de entrada plantea más semejanzas entre ellos que diferencias, puesto que tanto el ApS (Kendall, 1990) como las CdA (Elboj, Puigdellívol, Soler y Valls, 2006) consideran elemental la relación entre comunidad-centro y, además, señalan los beneficios pedagógicos que supone educar en el contexto comunitario. Finalizamos haciendo un balance, destacando la existencia de tensiones y conflictos que pueden dificultar el diálogo entre ambos, a la vez que se identifican notas comunes, que pensamos que pueden ayudar a lograr una misión que es compartida: la mejora escolar mediante la implicación comunitaria en el proceso educativo. 
CARMEN ÁLVAREZ ÁLVAREZ Y GONZALO SILIÓ SÁIZ EL APRENDIZAJE-SERVICIO Y LAS COMUNIDADES DE APRENDIZAJE: DOS PROYECTOS ESCOLARES INNOVADORES QUE SE ENRIQUECEN MUTUAMENTE

\section{DOS PROYECTOS APOYADOS EN CONCEPCIONES DIFERENTES}

El aprendizaje-servicio y las comunidades de aprendizaje constituyen dos proyectos educativos innovadores, relevantes y actuales en el marco escolar. El ApS es una metodología que fomenta la adquisición de valores cívicos (Puig, 2007) mediante la combinación del aprendizaje escolar con el servicio solidario protagonizado por el alumnado, presentándose como «una metodología educativa que permite la conexión entre el centro escolar y comunidad, una innovadora propuesta de incorporación del servicio voluntario en el aula, una herramienta para hacer posible el aprendizaje significativo y contextualizado de los aprendizajes curriculares» (Cieza, 2010: 130).

Por su parte, CdA es un proyecto de transformación de la escuela con el objetivo de luchar contra el fracaso escolar y conseguir que la sociedad de la información no excluya a ninguna persona, con especial énfasis en el alumnado más vulnerable, con la intención de "conseguir la utopía de aquella escuela o de la educación que todo el mundo quiera tener y, sobre todo, hacer realidad el sueño de que ningún niño ni niña quede marginado/a o etiquetado/a por la procedencia de su clase social, etnia, estatus económico, género, etc.» (Flecha y Puigvert, 2002: 2).

A continuación exploramos más ampliamente las bases teóricas de cada uno de ellos y su repercusión en nuestras escuelas.

\subsection{Aprendizaje-servicio}

El ApS es una práctica educativa habitual en muchos países llegando a estar presente en el 25\% de las escuelas de Educación Primaria y Secundaria de Estados Unidos (CNCS, 2011). También es una de las formas más comunes del servicio social obligatorio impuesto en el año 2006 en todos los centros de secundaria holandeses (Bekkers, 2009). Desde el Centro Latinoamericano de Aprendizaje y Servicio Solidario (CLAYss) aseguran que el ApS ha sido aplicado en miles de experiencias en todo el continente, siendo especialmente destacadas las de Argentina (el ApS es reconocido incluso en su ley educativa de 2007 en los artículos 32 y 123), Brasil, Uruguay y Chile. Así mismo, en los últimos años, las prácticas de ApS se han expandido por todo el mundo existiendo proyectos en Sudáfrica, India (Luna, 2010), Japón, Singapur, Tailandia y Oceanía (Silió Sáiz, 2010). Esta expansión global se debe en parte a la flexibilidad de aplicación del ApS y a la familiarización de los docentes con los componentes que lo forman (Puig, 2007). En nuestro país, donde existen más de 4 redes de promoción y 15 grupos promotores (destacan Zerbikas en el País Vasco y el Centro Promotor de Aprendizaje Servicio en Cataluña -Battle, 2013-), se pueden identificar experiencias en casi todo el Estado y en todas las etapas educativas.

Es fácil identificar que el ApS responde a un tipo de escuela alejado del modelo industrial basado en patrones bancarios (Freire, 1971), y que en cambio se acerca a un paradigma educativo que, aunque no es novedoso, sí es actual y acorde 
con las expectativas de nuestras sociedades actuales (Delors, 1996; Tedesco, 2000), donde la experiencia práctica y la búsqueda de desarrollo personal y social aparecen como protagonistas ineludibles. En esta línea, Rifkin (1996) ha asegurado que el ApS es un antídoto contra el aislamiento que experimentan los niños y jóvenes en todos sus contextos de vida, ya que al dárseles una oportunidad de participación profunda en la comunidad se los ayuda a desarrollar el sentido de la responsabilidad, desarrollando su autoestima y capacidad de liderazgo y, sobre todo, permitiendo que crezcan y florezcan el sentido de creatividad, iniciativa y empatía.

Si bien se aceptan ampliamente como bases fundamentales del ApS los planteamientos de Williams James y John Dewey (Puig y Palos, 2006) y especialmente de este último, sus teorías del aprendizaje basado en la experiencia (Dewey, 1995). Por otro lado, es común establecer relaciones entre el ApS con varias corrientes pedagógicas (Butin, 2003). Se ha escrito que Gandhi empleaba con frecuencia el ApS (Tapia, 2006). También se han establecido relaciones entre el Aps y Baden Powel, y con la pedagogía socialista del trabajo (Chanes, 2006; Trilla, 2009 en Tapia, 2006). Así mismo, hay varios trabajos que han relacionado el ApS con Aristóteles, Vigostsky, Piaget, Bandura o incluso Frankl, Freinet, Goleman o Gardner (Tapia, Gonzalez y Elicegui, 2005). En resumen, tanto las teorías del constructivismo (Furco, 2001) como de la Escuela Nueva (Tapia, González y Elicegui, 2005) son recogidas o se materializan con el ejercicio del ApS, ya que el aprendizaje significativo y la investigación-acción son sus piedras angulares. Como es cierto que las relaciones con sus bases teóricas son amplias y difieren en función de si se piensa en el ApS como filosofía, pedagogía o metodología (Jacoby, 2003). También hay relaciones estrechas con el discurso de Freire y de Habermas (Folgueiras y Martínez, 2009), especialmente al referirnos a sus conceptos relacionados con la acción dialógica (Freire, 1971) y la acción comunicativa (Habermas, 1992). Aspectos ampliamente relacionados con las CdA (Flecha, 1997) y ya señalados en Folgueiras y Martínez (2003). Unido esto al papel esencial que presenta la interacción con la comunidad en el ApS (Martínez-Odría, 2007) tanto que sin alteridad no podemos hablar de ApS, pues perdería la reciprocidad mutua entre el dar y recibir: el aprender y servir.

Como apunta Cieza (2010: 130), el ApS «enfatiza tanto el aprendizaje académico que se desarrolla en el aula como la realización de un servicio voluntario a favor de los problemas y necesidades reales detectadas en la comunidad próxima de forma que ambos (servicio y aprendizaje) se enriquecen mutuamente y forman un binomio inseparable». En palabras de Tapia (2001) clara y contundentemente: aprender sirve; y servir enseña, y al hacerlo de forma conjunta, se benefician las calidades y repercusiones de ambos (Furco, 2004). Por último, y como conclusión, es necesario señalar que son tres los componentes esenciales de una práctica de ApS (Puig y Palos, 2006; Tapia, 2004): 1) adquisición de aprendizajes recogidos en el currículum que el alumnado debe adquirir; 2) que los aprendizajes se adquieran mediante el desarrollo de servicio solidario sentido por la comunidad que lo recibe; 3) y que el alumnado tenga protagonismo en todo el proceso. 
CARMEN ÁLVAREZ ÁLVAREZ Y GONZALO SILIÓ SÁIZ

EL APRENDIZAJE-SERVICIO Y LAS COMUNIDADES DE APRENDIZAJE:

DOS PROYECTOS ESCOLARES INNOVADORES QUE SE ENRIQUECEN MUTUAMENTE

\subsection{Comunidades de aprendizaje}

Las CdA, learning communities, han demostrado su interés y valía en diferentes investigaciones nacionales e internacionales, siendo especialmente destacado el Proyecto integrado del VI Programa Marco de la Unión Europea INCLUD-ED (http:// creaub.info/included/), evidenciando su poder transformador en contextos educativos difíciles, logrando mejoras notables en aspectos tan importantes como los resultados escolares, la convivencia y la motivación.

Las CdA tienen unos antecedentes claros: a nivel internacional, el Programa de Desarrollo Escolar, el programa Éxito Para Todos y las Escuelas Aceleradas, y en España, el Centro de Educación de Personas Adultas de la Verneda de Sant Martí. En todos ellos se considera fundamental la participación de la comunidad en la escuela, tener altas expectativas de todo el alumnado (sin exclusiones) y promover el máximo aprendizaje para todas las personas implicadas, aspecto compartido con las CdA (Racionero y Serradel, 2005). Por ello, en CdA son aspectos clave la implicación de las familias en el centro educativo, la solidaridad entre todas las personas de la comunidad escolar y la inclusión educativa (Puigvert y Santacruz, 2006; Martínez y Niemelä, 2010; Molina y Holland, 2010).

Las CdA sientan sus bases teóricas en planteamientos aportados por la comunidad científica internacional, alimentándose de diversos campos del conocimiento (pedagogía, sociología, psicología, filosofía, etc.), como los planteamientos de Habermas (1987) en la Teoría de la Acción Comunicativa, de Freire (1970) en la Pedagogía del oprimido o de Vigotsky (1979) en El desarrollo de los procesos psicológicos superiores, entre otros, construyendo a su amparo un marco teórico con carácter multidisciplinar que permite fundamentar la acción didáctica: el aprendizaje dialógico (Flecha, 1997; Aubert, Flecha, García, Flecha y Racionero, 2008).

El aprendizaje dialógico es el eje teórico fundamental en CdA y se ha configurado a partir de la investigación de Flecha (1997) y el trabajo del equipo de investigación CREA (Centro de investigación en teorías y prácticas superadoras de desigualdades de la Universidad de Barcelona).

El aprendizaje dialógico considera que aprendemos a partir de la interacción comunitaria con otras personas generando acuerdos intersubjetivos entre las personas participantes (Aubert, Flecha, García, Flecha y Racionero, 2008). Según la concepción dialógica del aprendizaje, para aprender las personas necesitamos gran número de interacciones y lo más diversas posibles, apoyándose el diálogo en una relación de igualdad y no de poder, lo que significa que todas las personas tenemos conocimiento que aportar, reconociendo así la inteligencia cultural de todas las personas.

Todas las intervenciones que se basen en el aprendizaje dialógico deben estar guiadas por siete principios: la dimensión instrumental, la inteligencia cultural, la creación de sentido, la solidaridad, el diálogo igualitario, la igualdad de diferencias y la transformación (Flecha, 1997; Vargas y Flecha, 2000; Elboj, Puigdellívol, Soler y Valls, 2006). Todos ellos sirven de base teórica en el proceso de construcción de una CdA en un centro. Tras una fase de sensibilización del profesorado, toda la comunidad educativa del centro escolar sueña la escuela que desea, se 
forman comisiones mixtas para priorizar y hacer realidad los sueños de todas las personas y se inician prácticas de éxito en la escuela que contribuyen a reducir los índices de fracaso escolar (grupos interactivos, bibliotecas tutorizadas, tertulias literarias, formación de familiares, etc.).

Convenimos con los planteamientos de Guarro (2005) en que los rasgos que determinan estas CdA son:

a. Participación: referida a todos los agentes educativos implicados en la formación de tal forma que se optimicen recursos y se desarrollen nuevas estructuras organizativas.

b. Centralidad del aprendizaje: conseguir que todos desarrollen al máximo sus potencialidades es el objetivo fundamental. Para ello se debe reestructurar la organización de la escuela, aprovechando al máximo el tiempo de aprendizaje y planificando colectivamente la enseñanza.

c. Expectativas positivas: se parte de la consideración de altas expectativas del alumnado y de la apuesta por las capacidades que todos poseemos.

d. El progreso permanente: asumiendo la evaluación del proceso de tal forma que nos permita comprobar los aspectos positivos y las necesidades de mejora.

El proyecto de CdA lleva en funcionamiento desde el curso 1995-1996 en diversos centros de Educación Infantil, Primaria y Secundaria constituyendo una realidad en más de ciento noventa escuelas repartidas entre España e Iberoamérica. Desde que se transformó la primera escuela española en CdA, este proyecto no ha dejado de crecer y demostrar una y otra vez su enorme potencial para la mejora educativa y la disminución del fracaso escolar. Basta observar la página web del proyecto para ver su enorme difusión en España y su incipiente calado en otros países (http://utopiadream.info/ca/?page_id-1645) donde, además, es posible documentarse ampliamente sobre las bases teóricas y las diferentes prácticas de éxito que deben ser implementadas.

TABLA 1

Comparativa entre ApS y CdA.

\begin{tabular}{|c|c|c|}
\hline & APS & CDA \\
\hline $\begin{array}{c}\text { Fundamentos } \\
\text { teóricos }\end{array}$ & \begin{tabular}{|} 
- El aprendizaje se incrementa al \\
realizarse de forma experimen- \\
tal y vinculado a un servicio a la \\
comunidad (Ammon, Furco, Chi \\
y Middaugh, 2001; Dávila y Mora, \\
2007; Billig, 2003; Furco, 2007; \\
Garcia-Rodicio y Silió Sáiz, 2012).
\end{tabular} & $\begin{array}{l}\text { - Participación de todos los agen- } \\
\text { tes educativos implicados en la } \\
\text { formación. } \\
\text { - Centralidad del aprendizaje: con- } \\
\text { seguir que todos desarrollen al } \\
\text { máximo sus potencialidades es el } \\
\text { objetivo fundamental. }\end{array}$ \\
\hline
\end{tabular}


CARMEN ÁLVAREZ ÁLVAREZ Y GONZALO SILIÓ SÁIZ

EL APRENDIZAJE-SERVICIO Y LAS COMUNIDADES DE APRENDIZAJE: DOS PROYECTOS ESCOLARES INNOVADORES QUE SE ENRIQUECEN MUTUAMENTE

\begin{tabular}{|c|c|c|}
\hline & APS & $\mathrm{CDA}$ \\
\hline & $\begin{array}{l}\text { - El servicio solidario mejora al } \\
\text { vincularse a un aprendizaje aca- } \\
\text { démico (Tapia, 2001; Billig, Root } \\
\text { y Jesse, 2005; Martin, Neal, Kiels- } \\
\text { meier y Crossley, 2006). }\end{array}$ & $\begin{array}{l}\text { - Expectativas positivas: para todas } \\
\text { las personas, especialmente los } \\
\text { niños. } \\
\text { - El progreso permanente: eva- } \\
\text { luando los procesos y promo- } \\
\text { viendo la mejora (Guarro, 2005). }\end{array}$ \\
\hline Finalidades & $\begin{array}{l}\text { - Mejorar la significatividad de los } \\
\text { aprendizajes escolares (Dewey, } \\
\text { 1971; Freinet, 1967; Tonucci, } \\
\text { 1979 en Puig y otros, 2011). } \\
\text { - Provocar mejora o desarrollo } \\
\text { social (Conrad y Hedin, } 1989 \text { en } \\
\text { Furco y Billig, 2002; Argentina, } \\
\text { Ley de Educación Nacional, arts. } \\
32 \text { y 123, 2007). } \\
\text { - Educar en valores de ciudadanía } \\
\text { (Puig, 2010). }\end{array}$ & 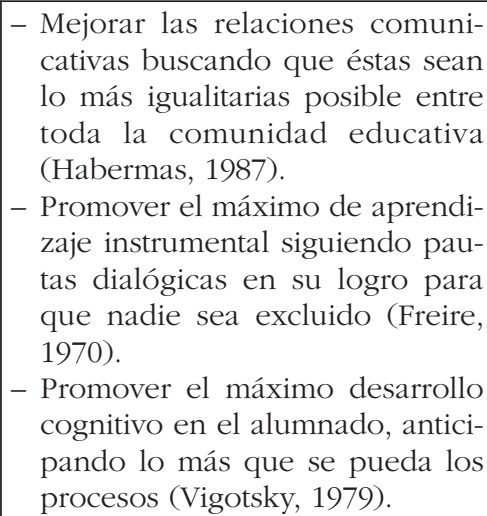 \\
\hline $\begin{array}{c}\text { Prácticas de } \\
\text { enseñanza }\end{array}$ & $\begin{array}{l}\text { Actividades prácticas vinculadas } \\
\text { a satisfacer una necesidad sentida } \\
\text { de una comunidad mediante el tra- } \\
\text { bajo y los conocimientos que debe } \\
\text { adquirir el alumnado. Se caracteri- } \\
\text { zan por: el papel activo del alumno } \\
\text { en todo el desarrollo; la planifi- } \\
\text { cación de los aprendizajes que se } \\
\text { pretende suscitar con la actividad; } \\
\text { la conexión con el currículo de } \\
\text { los mismos; la reflexión sobre lo } \\
\text { acontecido durante la actividad; } \\
\text { el respeto y reconocimiento de } \\
\text { la diversidad como una fortaleza } \\
\text { y la capacidad para generar alian- } \\
\text { zas entre personas e instituciones } \\
\text { (Furco y Billig, 2002; NYLC, 2008; } \\
\text { Puig, Batlle, Bosch y Palos, 2007; } \\
\text { Tapia, 2006). }\end{array}$ & $\begin{array}{l}\text { Prácticas de éxito avaladas por la } \\
\text { comunidad científica internacional, } \\
\text { que han demostrado su poder ins- } \\
\text { tructivo y formativo en todos los } \\
\text { contextos donde se han desarro- } \\
\text { llado. Las actuaciones educativas } \\
\text { de éxito más destacadas hoy día } \\
\text { son: } \\
\text { - los grupos interactivos } \\
\text { - las bibliotecas tutorizadas } \\
\text { - las tertulias dialógicas (literarias } \\
\text { curriculares, de artes, musicales, } \\
\text { para profesorado...) } \\
\text { - la formación de familiares } \\
\text { - entre otras. }\end{array}$ \\
\hline $\begin{array}{l}\text { Agentes } \\
\text { implicados }\end{array}$ & $\begin{array}{l}\text { Docentes, alumnado, receptores } \\
\text { del servicio solidario. }\end{array}$ & $\begin{array}{l}\text { Docentes, alumnado, equipos } \\
\text { directivos, familiares del alum- } \\
\text { nado y comunidad educativa de la } \\
\text { escuela en general. }\end{array}$ \\
\hline
\end{tabular}

Fuente: Elaboración propia. 


\section{3. ¿Dos proyectos alternativos?}

Una vez presentadas las bases teóricas del ApS y de las CdA es evidente que entre ambos proyectos hay diferencias, pudiendo parecer que ambos constituyen dos proyectos alternativos y excluyentes: si existe el uno, no se da el otro. Destacaremos tres aspectos que nos parecen fundamentales:

1. CdA prioriza el aprendizaje para evitar posibles situaciones de vulnerabilidad escolar y favorecer el éxito académico del alumnado, considerando que la educación en valores se produce en el centro a través de la participación solidaria de la comunidad escolar, logrando que el aprendizaje se dé en condiciones de solidaridad. Por su parte, el ApS introduce de forma equilibrada un componente de servicio solidario en los aprendizajes curriculares de los alumnos con el objetivo de que sus prácticas curriculares puedan ocasionar una mejora social en la comunidad. A la vez, dicha experiencia pedagógica práctica genera un contexto idóneo para resaltar la educación en valores especialmente del alumnado y también de la comunidad educativa (Álvarez y Larrinaga, 2012).

2. Respecto a la concepción de la solidaridad, CdA maneja una muy ligada al compromiso de la comunidad educativa del centro educativo en sentido amplio: trata de incorporar a la escuela personas de muy variados perfiles, de tal manera que el entorno participa de la vida del centro, que pasa a configurarse como un espacio aglutinador de todas las actividades de aprendizaje y solidaridad, que vivirá el alumnado en su día a día. El profesorado y el alumnado a su vez también viven esta solidaridad porque el centro está en proceso de transformación permanente, haciendo realidad este principio. Por su parte, el ApS persigue y provoca que sea el alumnado quien sienta, practique y asuma la solidaridad en el proyecto. Pero, a su vez, en los proyectos de ApS de mayor calidad se produce una doble reciprocidad. A la vez que se protagoniza un servicio, se recibe el beneficio de otro. Sí es verdad que siempre el ejercicio de la solidaridad va ligado a un aprendizaje curricular, dentro del ApS y por lo tanto a un grupo de educandos.

3. En ambos proyectos, la idea de voluntariado y solidaridad es central, pero en el ApS se pretende que sobre todo sean los estudiantes: infantes y adolescentes, quienes actúen solidariamente en su entorno social. Es cierto que el centro o profesorado que desarrolle dicha metodología está poniendo en valor y acción la solidaridad como parte de su responsabilidad educativa. Pero sobre quienes recae el peso de la acción solidaria es sobre los estudiantes. Mientras, en CdA, se considera que la acción solidaria la desarrollan los niños entre sí en el aula y fuera de ella, las personas de la comunidad que se vinculan al centro, el profesorado entre sí, etc., ampliando los marcos de acción educativa y social. 
CARMEN ÁLVAREZ ÁLVAREZ Y GONZALO SILIÓ SÁIZ EL APRENDIZAJE-SERVICIO Y LAS COMUNIDADES DE APRENDIZAJE: DOS PROYECTOS ESCOLARES INNOVADORES QUE SE ENRIQUECEN MUTUAMENTE

Asimismo, hay una diferencia significativa entre ambos a la hora de su implementación. En CdA se requiere la aprobación del claustro de profesorado, Consejo Escolar, Equipo directivo, AMPA y comunidad educativa escolar para iniciar el proyecto, en el ApS el cambio se produce en la práctica cotidiana entre docentes, en una relación informal y horizontal que se contagia.

\section{DOS PROYECTOS QUE RELACIONAN LAS ESCUELAS Y SU COMUNIDAD EDUCATIVA}

Pese a que desde el punto de vista teórico hay sensibles diferencias entre el ApS y las CdA en sus fundamentos teóricos, en sus prácticas puede ser difícil deslindar qué práctica pertenece a cada proyecto en numerosas ocasiones. Ello es así porque en ambos hay pilares fundamentales compartidos: (1) el entorno como marco superador del quehacer del centro, (2) el papel de la comunidad educativa y el voluntariado y (3) la solidaridad social como principio. En resumen y parafraseando a Dewey, ambas propuestas van más allá de «educar para la vida, sino que intentan educar en la vida». En definitiva, lo que ocurre en los centros que desarrollan estas iniciativas es lo siguiente:

\subsection{Aprendizaje-servicio}

Cuando un docente decide introducir el ApS está buscando y propiciando: 1) que su responsabilidad profesional vaya más allá de su aula y su centro; 2) que las oportunidades de aprendizaje de los educandos sean más significativas y por tanto de mayor calidad; 3) que el esfuerzo y dedicación de sus alumnos provoque una mejora social además de personal, y 4) que el alumnado y él mismo tengan la posibilidad de ejercitar su ciudadanía de forma responsable aprovechando la oportunidad para ejercer una ciudadanía crítica. Por ello, al implementar prácticas de ApS ocurre que:

- Se propicia la aparición de otras voces en el aula, además de la del alumnado, que se convierte en protagonista (Billig, 2007; Martínez-Odría, 2007), y aparecen los miembros de la comunidad como agentes educativos.

- Las competencias se practican y pasan de ser un objetivo teórico a alcanzar a ser un vehículo real donde asumir y responsabilizarse de la ejecución del servicio solidario.

- La práctica docente pasa a ser colaborativa, pues es frecuente que sean varios docentes los implicados en los proyectos, o alguna otra persona de la contraparte (quienes reciben el servicio), asumiendo responsabilidades educativas. Asimismo, el contexto social y sus integrantes pasan a ser propulsores de aprendizajes.

- El centro escolar se abre porosamente a la realidad social que le rodea o le afecta. 
- El ApS se contagia horizontalmente, como lluvia fina al resto de profesionales, siendo en ocasiones los propios estudiantes quienes reclaman el ejercicio de dicha práctica.

La introducción del ApS en un grupo, centro o comunidad concreta genera casi automáticamente cambios organizacionales para facilitar el desarrollo de nuevos proyectos y para incrementar la calidad de los mismos. Por ejemplo, es habitual que varios proyectos se amplíen pasando a ser las familias y otros grupos también protagonistas de las prácticas.

\subsection{Comunidades de aprendizaje}

Las escuelas que se transforman en comunidades de aprendizaje se abren a la comunidad vinculando al centro educativo y su entorno próximo de múltiples maneras, a través de diversas prácticas educativas:

- El sueño conjunto de la comunidad educativa de la escuela deseada, la priorización de acciones a emprender y el trabajo para su logro a través de comisiones mixtas formadas por diferentes agentes de la comunidad educativa, buscando su diversidad interna.

- En las aulas se hacen grupos interactivos, que son una forma de organización escolar pensada para acelerar el aprendizaje que dispone al alumnado en grupos heterogéneos (en nivel de conocimientos, sexo, etnia, cultura, etc.) compuestos por el mismo número de alumnos/as para trabajar con el apoyo de un voluntario de la comunidad actividades de áreas instrumentales básicas durante una parte de la jornada escolar (generalmente una hora y media semanal).

- También se hacen bibliotecas escolares y salas de informática tutorizadas, que son una forma de aprovechar al máximo los recursos del centro para que el alumnado pueda realizar sus deberes en horario extraescolar con apoyos externos (personas voluntarias de la comunidad, profesorado voluntario, etc.), así como beneficiar de sus servicios a las familias y personas del contexto próximo al centro.

- Las familias del alumnado reciben formación en el centro en los campos de ellas mismas decidan: informática, idiomas, formación para conseguir el carnet de conducir, etc.

- En las CdA se pone en marcha el modelo dialógico de prevención de conflictos para generar en las escuelas el mejor clima de convivencia, asumiendo el reto de que en sus centros la violencia desaparezca por completo.

- En los centros que son CdA es posible reconocer la solidaridad social como un principio articulador de las acciones educativas de la escuela. 
CARMEN ÁLVAREZ ÁLVAREZ Y GONZALO SILIÓ SÁIZ EL APRENDIZAJE-SERVICIO Y LAS COMUNIDADES DE APRENDIZAJE: DOS PROYECTOS ESCOLARES INNOVADORES QUE SE ENRIQUECEN MUTUAMENTE

\section{3. ¿Dos proyectos complementarios?}

Ya hemos expresado con anterioridad que ambas propuestas pedagógicas: 1) comparten bases teóricas aunque discrepen en algunos postulados; y 2) que la relación entre la comunidad-centro, el aprovechamiento de la realidad social como fuente de aprendizaje y la mejora de la misma como objetivo son comunes en ambos proyectos. Ahora, podemos plantearnos: ¿son proyectos complementarios? Nuestra visión es que ninguno de los dos proyectos necesita del otro para su desarrollo, pero su posible relación puede beneficiar la calidad de los mismos y mejorarlos, nunca perjudicarlos.

En una CdA podrían desarrollarse proyectos de ApS. Es más, el ApS podría ofrecer un lugar idóneo para ejercer la reflexión crítica que fundamentan a las CdA. Asimismo, los centros que desarrollan proyectos de ApS podrían incorporar prácticas de las CdA, e incluso, a través de ellas, promover la transformación de los centros en CdA. Existen precedentes en esta línea: Furco (2010) investigó sobre "centros que aprenden" y comunidades educativas centradas en el servicio a la comunidad y en el estudio social de la misma. Por su parte, Cecchi (2006) ha resaltado tanto la intencionalidad pedagógica y la intencionalidad solidaria como matices determinantes de una institución que enarbole el ApS. Es decir, incide en la reflexión filosófica sobre para qué sirve un centro que aplica ApS, debate que es compartido por algunos autores que reclaman una visión filosófica del ApS (Puig, 2007), que a su vez es una reflexión que abordan al inicio las CdA.

Por otro lado, CdA, busca y logra promover la transformación del centro educativo y su entorno, provocando la mejora educativa de los niños y de sus comunidades, realizando un servicio educativo y social que va más allá de la vida ordinaria de la escuela (Puigvert y Santacruz, 2006): muchas familias se motivan a crear cooperativas o estudiar tras participar un tiempo como voluntarias en grupos interactivos, se empiezan a interesar por la educación de sus hijos o se implican más que antes de ser CdA, familias que aprenden a leer y escribir o se sacan el título de Secundaria o estudian idiomas o sacan el carnet de conducir... son realidad cada día en el proyecto. Esta reciprocidad mutua entre comunidad y escuela está presente también en el ApS. Sobre todo en aquellos proyectos considerados ejemplares se observa un beneficio mutuo para el centro y la comunidad (Furco, 2002). Podríamos decir que tanto el ApS como las CdA comparten y disfrutan de una naturaleza recíproca del servicio solidario (D’Arlach, Sánchez y Feuer, 2009: 6) y un profundo sentido prosocial (Cecchi, 2006: 2-4). También sucede en CdA que algunos antiguos alumnos de las escuelas transformadas deciden hacerse voluntarios en los centros en su etapa de Educación Secundaria, demostrando haber valorado la acción solidaria recibida de la comunidad y devolviéndola a su escuela. Por su parte, los estudiantes que participaron en proyectos de ApS recomiendan y animan a sus compañeros a formar parte de ellos (Billig y Meyer, 2002; Billig, Meyer y Hofschire, 2003), lo que denota la satisfacción del alumnado con ambos proyectos y la prociudadanía que generan ambas prácticas. 
4. El APS Y LAS CDA: DOS PROYECTOS INNOVADORES QUE SE ENRIQUECEN MUTUAMENTE

Entre ambos proyectos hemos visto que se producen sensibles diferencias, sobre todo en lo que se refiere a sus sustratos teóricos, pero en las prácticas reales en la enseñanza que se desarrollan en centros educativos hay cierta sintonía, sobre todo en la relación tan estrecha que cultivan ambos proyectos con la comunidad escolar, su confianza en las prácticas de voluntariado, su preocupación por el aprendizaje y la solidaridad. Así mismo hay un elemento central en ambas realidades: el sentir y propiciar la acción social del alumnado como parte inherente de su proceso formativo como ciudadano crítico y activo con capacidad de cambio y mejora en su comunidad.

Por todo ello, concluimos que el ApS y las CdA constituyen dos proyectos innovadores que se enriquecen mutuamente: para ambos son fundamentales la aproximación escuela-comunidad, el voluntariado y la solidaridad y el papel activo y ciudadano de los estudiantes.

Como plantea Apple (2005), sólo en contacto con la realidad se formará a los niños en el desarrollo de actitudes reflexivas y comprometidas con sus territorios, aspecto que cultivan tanto el ApS como las CdA. Ambos proyectos también inciden en la mejora de la comunidad y fortalecen su tejido social (Puig y otros, 2007), promoviendo cambios en las mentalidades y las prácticas de los diferentes agentes implicados.

Respecto a los aprendizajes académicos, el impacto de ambos proyectos ha incrementado la competencia de los estudiantes (Furco, 2003; Billig, 2007; Weah, 2007; Vieira y Puigdellívol, 2013). También a nivel de convivencia y motivación por la educación ambos proyectos ofrecen mejoras (Luna, 2010; Elboj y Niemelä, 2010; Martínez y Niemelä, 2010).

Existen y existirán tensiones y conflictos entre ambos proyectos pero en el futuro cada vez será más posible el diálogo: ApS y CdA son proyectos innovadores muy ricos que han ofrecido alternativas educativas de interés. Complementándose y colaborando solidariamente ambos proyectos ampliarán sus marcos teóricos y prácticos, aprovechando las potencialidades y aminorando las diferencias entre ellos. Las diferencias se dan más en las fundamentaciones teóricas que en la práctica docente y social, donde ambos son complementarios y sumativos.

\section{REFERENCIAS BIBLIOGRÁFICAS}

Álvarez, C. y Larrinaga, A. (2012). Iniciando grupos interactivos en la comunidad cántabra. Aula de Innovación Pedagógica, 210, 53-57.

Ammon, M. S.; Furco, A.; Chi, B. y Middaugh, E. (2002). Service Learning in California: A Profile of the CalServe Service-Learning Partnerships (1997-2000). Berkeley: University of California.

Aubert, A.; Flecha, A.; García, C.; Flecha, R. y Racionero, S. (2008). Aprendizaje dialógico en la Sociedad de la Información. Barcelona: Hipatia. 
CARMEN ÁLVAREZ ÁLVAREZ Y GONZALO SILIÓ SÁIZ EL APRENDIZAJE-SERVICIO Y LAS COMUNIDADES DE APRENDIZAJE: DOS PROYECTOS ESCOLARES INNOVADORES QUE SE ENRIQUECEN MUTUAMENTE

Bekkers, R. (2009). A New National Service Learning Program in the Netherlands: Preliminary Evidence. Forum21 - European Journal on Child and Youth Research, 3, 62-6.

Billig, S. H. y Brodersen, R. M. (2007). Case Studies of Effective Practices in the Partnership in Character Education Project: Evaluation for the School District of Philadelphia. Denver: RMC Research Corporation.

Billig, S. H. y Conrad, J. (1997). An evaluation of the New Hmapshire Service-Learning and Educational Reform Project. Denver: RMC Research Corporation.

Billig, S. H.; Dan, J.; Lee, C. y Kristin, K. (1999). An Evaluation of Jefferson County School District's School-to-Career Partnership Program. Denver: RMC Research Corporation.

Billig, S. H. y Meyer, S. (2002). Evaluation of the Hawaiian Studies Program at Waianae High School for CREDE. Denver, CO: RMC Research Corporation.

Billig, S. H.; Meyer, S. y Hofschire, L. (2003). Evaluation of Center for Research on Education, Diversity, and Excellence demonstration site, the Hawaiian Studies Program at Waianae High School. Denver, CO: RMC Research Corporation.

Billig, S. H.; Root, S. y Jesse, D. (2005). The Impact of Participation in Service-Learning on High School Students' Civic Engagement. Denver: RMC Research Corporation.

Butin, D. (2003). Of what use is it? Multiple conceptualizations of service learning within education. Teachers College Record, 105 (9), 1674-1692.

Cecchi, N. (2006). Aprendizaje Servicio en Educación Superior. La experiencia latinoamericana. En Seminario Internacional Responsabilidad Social Universitaria: Aprendizaje Servicio. Universidad Central de Venezuela. Descargado el 5 de diciembre de 2013 en: http://www.documentacion.edex.es/docs/0406CECapr.pdf.

Cieza García, J. A. (2010). El compromiso y la participación comunitaria de los centros escolares. Un nuevo espacio-tiempo de intervención socioeducativa. Revista Interuniversitaria de Pedagogía Social, 17, 123-136.

Conrad, D. y Hedin, D. (1980). Executive Summary of the Final Report of the Experiential Education Evaluation Project. Minneapolis: University of Minnesota.

Corporation for National \& Community Service, CNCS (2008). Community Service and ServiceLearning in America's Schools. Descargado el 1 de julio de 2011 en: http://www.nationalservice.gov/about/role_impact/performance_research.asp.

D'Arlach, L.; Sánchez, B. y Feuer, R. (2009). Voices from the community: a case for reciprocity in Service-Learning. Michigan Journal of Community Service Learning. Descargado el 5 de diciembre de 2013. http://quod.lib.umich.edu/cgi/p/pod/dod$\mathrm{idx} / 3239521.0016 .101 . p d f ? c-m j c s l ; i d n o-3239521.0016 .101$.

Dávila, A. y Mora, M. (2007). Civic Engagement and High School Academic Progress: An Analysis Using NELS Data. College Park, MD: University of Maryland School of Public Policy, Center for Information and Research on Civic Learning and Engagement (CIRCLE).

Deans, T. (1999). Service-learning in two keys: Paulo Freire's critical pedagogy in relation to John Dewey's pragmatism. Michigan Journal of community service learning, 15-29.

Dewey, J. (1995). Democracy and Education. New York: Free Press.

Elboj, C. y Niemela, R. (2010). Sub-communities of mutual learners in the classroom: the case of Interactive groups. Revista de Psicodidáctica, 15 (2), 177-189.

Elboj, C.; Puigdellívol, I.; Soler, M. y Valls, R. (2006). Comunidades de aprendizaje. Transformar la educación. Barcelona: Graó.

Flecha, J. R. (1997). Compartiendo palabras. El aprendizaje de las personas adultas a través del diálogo. Barcelona: Paidós. 
Flecha, J. R. y Puigvert, L. (2002). Las comunidades de aprendizaje: una apuesta por la igualdad educativa. REXE, 1, 11-20.

Folgueiras, P. y Martínez, M. (2009). El desarrollo de competencias en la Universidad a través del Aprendizaje y Servicio Solidario. Revista Interamericana de Educación y Democracia, 2 (1). Descargado el 8 de diciembre de 2013. http://www.riedijed.org/spanish/ articulo.php?idRevista-9yidArticulo-28.

Freire, P. (1971). La educación como práctica de la libertad. Bogotá: Siglo XXI.

Furco, A. (2003). Issues of definition and program diversity in the study of service learning. En S. H. Billig (Ed.). Studying service-learning. London: Lawrence Erlbaum Publishing Company.

Furco, A. (2007). Role of Service-Learning in Enhancing Student Achievement. Descargado el 23 de abril de 2014 en: http://www.servicelearningtexas.org/_documents/Impacts_ Of_SL_on_Participating_K-12_Students_FS_Short_Final_Mar08-1.pdf.

Furco, A. (2010). The community as a resource for learning: An analysis of academic servicelearning in primary and secondary education. En H. Dummont, D. Istance y F. Benavides (Eds.). The nature of learning. Using research to inspire practice (pp. 227-248). Paris: OECD.

García Pérez, A. (2012). La respuesta a las necesidades educativas en las Comunidades de Aprendizaje. Greenwich: Information Age Publishing.

García-Rodicio, H. y Silió-Sáiz, G. (2012). Tomando la temperatura al Aprendizaje-Servicio. ¿Qué procesos de aprendizaje, fríos y cálidos, promueve? Revista Iberoamericana de Educación, 60 (2), 1-11. Descargado el 5 de marzo de 2014. http://www.rieoei.org/ deloslectores/4902Garcia-Rodicio.pdf.

Guarro, A. (2005). La transformación democrática de la cultura escolar: Una respuesta justa a las necesidades del alumnado de zonas desfavorecidas. Profesorado, Revista de Currículum y Formación del Profesorado, 1.

Jacoby, B. y otros (2003). Building Partnerships for Service-Learning. San Francisco: John Wiley y Sons.

Kendall, J. (1990). Using students effectively in your organization. En J. Kendall and Associates (Eds.). Combining service and learning: A resource book for community and public service, vol. II (pp. 219-227). Raleigh: National Society for Internships and Experiential Education.

Kingsland, S. F.; Richards, M. y Coleman, L. (1995). A Status Report for KIDSNET, Year One, 1994-1995. Portland: University of Southern Maine.

Kinsley, C. (1997). Service Learning: A Process to Connect Learning and Living. In ServiceLearning: Leaving Footprints on the Planet. National Association of Secondary School Principals Bulletin, 1-7. http://dx.doi.org/10.1177/019263659708159102

Luna, E. (2010). Del centro educativo a la comunidad: Un programa de aprendizaje-servicio para el desarrollo de la ciudadanía activa. Tesis doctoral. Barcelona: Universidad de Barcelona.

Martin, S.; Neal, M.; Kielsmeier, J. y Crossley, A. (2006). The impact of service learning on transitions to adulthood. Growing to Greatness 2006. St. Paul: National Youth leadership council.

Martín, X.; Rubio, L.; Batlle, C. y Puig, J. M. (2010). Prácticas de ciudadanía. Diez experiencias de aprendizaje servicio. Barcelona: Octaedro.

Martínez, B. y Niemelä, R. (2010). Formas de implicación de las familias hacia el éxito educativo. Revista Educación y Pedagogía, 22 (56), 69-77. 
CARMEN ÁLVAREZ ÁLVAREZ Y GONZALO SILIÓ SÁIZ EL APRENDIZAJE-SERVICIO Y LAS COMUNIDADES DE APRENDIZAJE: DOS PROYECTOS ESCOLARES INNOVADORES QUE SE ENRIQUECEN MUTUAMENTE

Martínez, M. (Ed.) (2010). Aprendizaje servicio y responsabilidad social de las Universidades. Barcelona: ICE-Octaedro.

Martínez-Odría, A. (2007). Service-Learning o Aprendizaje-Servicio. La apertura de la escuela a la comunidad local como propuesta de educación para la ciudadanía. Bordón, 59 (4), 611-626.

Melchior, A. (1999). Summary Report: National Evaluation of Learn and Serve America. Waltham: Center for Human Resources, Brandeis University.

Molina, S. y Holland, C. (2010). Educación especial e inclusión: aportaciones desde la investigación. Revista Educación y Pedagogía, 22, 56.

Puig, J. (Coord.) (2011). Aprendizaje servicio. Aula de Innovación Educativa, 203-204, 10-65.

Puig Rovira, J. y Batlle, R. (2007). Aprendizaje-Servicio. Educar para la ciudadanía. Barcelona: Octaedro.

Puig, J. M. y Palos, J. (2006). Rasgos pedagógicos del aprendizaje-servicio. Cuadernos de Pedagogía, 357, 60-63.

Puigvert, L. y Santacruz, I. (2006). La transformación de centros educativos en comunidades de aprendizaje. Calidad para todas y todos. Revista de Educación, 339, 169-176.

Racionero, S. y Serradel, O. (2005). Antecedentes de las comunidades de aprendizaje. Educar, 35, 29-39.

Rifkin, J (1996). El fin del trabajo. Barcelona: Paidós Ibérica.

Rubio, L. (2009). El aprendizaje en el aprendizaje servicio. En J. M. Puig (Coord.). Aprendizaje-servicio. Educación y compromiso cívico (pp. 91-106). Barcelona: Graó.

Speck, B. W. y Hoppe, S. L. (2004). Service-Learning: History, Theory and Issues. Westport, Conn: Praeger Publishers.

Tapia, M. N. (2006). Aprendizaje y servicio solidario. Buenos Aires: Ciudad Nueva.

Tapia, M. N.; González, A. y Elicegui, P. (2005). Aprendizaje y servicio solidario en las escuelas argentinas: una visión descriptiva a partir de las experiencias presentadas al Premio Presidencial Escuelas Solidarias (2000-2001). Washington University: Global Service Institute. Descargado el 5 de diciembre de 2013. http://gwbweb.wustl.edu/csd/ service/SRGP_CLAYSS.htm.

Valls, R. (2006). Comunidades de aprendizaje: participación democrática y transformación social desde el aprendizaje dialógico. Actas del Primer Congreso Iberoamericano de Pedagogía Social.

Vargas, J. y Flecha, J. R. (2000). El aprendizaje dialógico como «experto» en resolución de conflictos. Contextos Educativos, 3, 81-88.

Vieira, L. y Puigdellívol, I. (2013). ¿Voluntarios dentro del aula? El rol del voluntariado en "Comunidades de Aprendizaje». REXE, 12 (4), 37-55.

VV. AA. (2010). Monográfico: Comunidades de aprendizaje. Revista Interuniversitaria de Formación del Profesorado, 67, 24 (1).

Weah, W. (2007). Toward research-based standards for k-12 service-learning. In Growing To Greatness 2007: The state of service learning. Descargado el 15 de enero de 2008 de: http://www.nylc.org/rc_downloaddetail.cfm?emoid-14:701.

Weiler, D.; LaGoy, A.; Crane, E. y Rovner, A. (1998). An Evaluation of K-12 Service-Learning in California: Phase II Final Report. Emeryville, CA: RPP International with the Search Institute. 